\title{
CDISC Protocol Entities Study Protocol Attribute Terminology
}

National Cancer Institute

\section{Source}

National Cancer Institute. CDISC Protocol Entities Study Protocol Attribute Terminology. NCl Thesaurus. Code C132309.

Terminology associated with the protocol entity study protocol attribute codelist of the Clinical Data Interchange Standards Consortium (CDISC). 